\title{
Role of Beclin I expression in patients with hepatocellular carcinoma: a meta-analysis
}

This article was published in the following Dove Press journal:

OncoTargets and Therapy

\author{
Chaojie Liang ${ }^{1, *}$ \\ Wei $\mathrm{Li}^{1, *}$ \\ Hua $\mathrm{Ge}^{1}$ \\ Kaitong Zhang ${ }^{2}$ \\ Guangming $\mathrm{Li}^{\prime}$ \\ Jixiang $\mathrm{Wu}^{\prime}$ \\ 'Department of General Surgery, \\ Beijing Tongren Hospital, Capital \\ Medical University, Beijing, People's \\ Republic of China; ${ }^{2}$ Department \\ of Tumor Center, Beijing Tongren \\ Hospital, Capital Medical University, \\ Beijing, People's Republic of China \\ *These authors contributed equally \\ to this work
}

Background and aim: Beclin1 has been reported as a vital marker for a number of malignant tumors. However, the role of Beclin1 in hepatocellular carcinoma (HCC) remains inconclusive. Thus, we conducted a meta-analysis to assess the correlation between Beclin1 and its clinicopathological and prognostic values in HCC.

Methods: PubMed, Cochrane Library, Web of Science, EMBASE, Chinese CNKI, and Chinese WanFang databases were searched for published articles on Beclin1 expression in hepatocellular tissues. Standard-compliant articles were screened using the Newcastle-Ottawa Scale for strict quality control of the literature. The correlation of Beclin1 expression with the clinicopathological features and survival outcomes was analyzed. Pooled odds ratios and hazard ratios with 95\% confidence intervals were calculated using STATA14.2.

Results: Eleven articles with 1,279 patients were included in this meta-analysis. Positive Beclin1 expression was found to be correlated with alpha fetoprotein, liver cirrhosis, and vascular invasion, but not with gender, age, HBsAg, size of tumor, number of tumors, differentiation, and TNM stage. Positive Beclin1 expression was also associated with favorable 5-year overall survival and disease-free survival rates.

Conclusion: Our meta-analysis indicated that positive Beclin1 expression was negatively related to alpha fetoprotein, liver cirrhosis, and vascular invasion in HCC. Beclin1 could be used as a prognostic biomarker for HCC.

Keywords: Beclin1, hepatocellular carcinoma, prognosis, meta-analysis

\section{Introduction}

Primary liver cancer is one of the most common malignancies in the digestive system and it has a poor prognosis. Hepatocellular carcinoma (HCC) comprises about 90\% of all types of primary liver cancer and is the third most common cause of cancerinduced mortality. ${ }^{1}$ The Asia-Pacific region has a high incidence of liver cancer, with People's Republic of China accounting for more than $50 \%$ of new cases of liver cancer worldwide annually. ${ }^{2}$ Currently, treatment for liver cancer is mainly based on surgery, transarterial chemoembolization, chemotherapy, sorafenib-targeted drug therapy, radiofrequency, and so on. These comprehensive treatment measures significantly improve the cure rate, effectively extending patients' life time. ${ }^{3}$ However, primary liver cancer is prone to early metastasis, which when combined with low surgical resection and postoperative metastasis and recurrence, greatly affect the efficacy of treatment. ${ }^{4,5}$ Numerous patients miss the opportunity of surgical treatment because of the lack of specific means for early detection and early treatment of liver cancer. Thus, a specific index of early predictions of liver cancer and the pathogenesis of HCC have recently drawn research interest. 
Changes in autophagic activation have been associated with the progression and development of tumors. ${ }^{6}$ Beclin 1 , which is located on the long arm of human chromosome 17 and about $150 \mathrm{~kb}$ in length, is a key protein that regulates autophagy and is regarded as a hallmark of autophagy initiation. Beclin1 plays an important role in the development and progression of various human malignancies. ${ }^{7}$ Beclin1 has been identified as a reliable biomarker in many cancers, such as gastric cancer, ${ }^{8}$ colorectal cancer, ${ }^{9}$ cervical cancer, ${ }^{10}$ and breast cancer, ${ }^{11}$ and it has been related to the clinical features and prognosis of tumors. A meta-analysis of Beclin1 and gastric cancer has shown that Beclin1 expression is negatively correlated with tumor differentiation and TNM staging. ${ }^{12}$ By contrast, another meta-analysis has demonstrated that Beclin1 expression is positively associated with tumor metastasis, poor prognosis, and overall survival (OS) in colorectal cancer. ${ }^{13}$ Beclin 1 expression has also been found to be poorly expressed in $\mathrm{HCC}$; however, its association with clinicopathological characteristics and prognostic value remains inconclusive. Qiu et a ${ }^{14}$ have indicated that Beclin1 is poorly expressed in HCC, relative to the adjacent tissue; positive Beclin1 expression has indicated a better prognosis. Notably, Wu et $\mathrm{al}^{15}$ have demonstrated that Beclin1 expression is not related to clinical features and prognosis. Osman et $\mathrm{a}^{16}$ have found that Beclin1 expression is only associated with HBsAg; therefore, a meta-analysis needs to be conducted to explore the relationship between Beclin1 expression and its clinicopathological characteristics and prognostic value in HCC.

\section{Methods}

\section{Search strategy}

Computer retrieval of commonly used biomedical databases, including Pubmed, Cochrane Library, Web of Science, EMBASE, Chinese CNKI, and Chinese WanFang, was conducted, and the search ended on September 1, 2017. The key words included the following: "beclin1" or "beclin-1" or "Atg6" and "hepatocellular carcinoma" or "hepatic tumor" or "liver tumor" or "hepatic cancer" or "liver cancer" or "HCC."

\section{Eligibility criteria}

A study was included in the meta-analysis if all of the following inclusion criteria were met: 1) availability of the full text of the article; 2) the patients in the literature had a definitive diagnosis of HCC and clinicopathological or survival information was included in the article; and 3) determination of Beclin1 expression by immunohistochemistry (IHC).
Studies were excluded if the case or control sources were unclear, and the pathological diagnosis was unclear. Republished studies using the same data source included only those with higher methodological quality.

\section{Data extraction}

All data were extracted, including the name of the first author, publication year, number of patients, region of origin, patient characteristics, and hazard ratios (HRs) with $95 \%$ confidence interval (CI) for OS, by 2 investigators (Chaojie Liang and Hua Ge), independently. Newcastle-Ottawa Scale (NOS) was used to evaluate the quality of the articles. Discrepancies were resolved by discussion, re-extraction, or third-party adjudication.

\section{Quality assessment}

We used NOS to assess the quality of the included articles, and articles were considered of good quality when the NOS score $\geq 6$ and of poor quality when the NOS score $\leq 5$. All articles included in this meta-analysis were of high quality.

\section{Statistical analysis}

Pooled odds ratios (ORs) with 95\% CIs were used to assess the association between positive Beclin1 expression and clinicopathological features calculated using STATA 14.2 (StataCorp LLC, College Station, TX, USA). The $\chi^{2}$-based $Q$-test and $I^{2}$ test were used to evaluate the heterogeneity between the studies. The heterogeneity was considered significant when $I^{2}$ values $>50 \%$ and PHet $\geq 0.1$. Random- or fixed-effects model was used in accordance with the results of heterogeneity analysis, and subgroup analysis by sample size or the NOS score was calculated to analyze the source of heterogeneity. We used Engauge Digitizer 10.0 (GitHub, Inc., San Francisco, CA, USA) to extract the survival data from a Kaplan-Meier curve in the articles. A favorable prognosis in the positive Beclin1 expression group was considered when HR $<1$ and if the $95 \%$ CI did not cross 1 . Potential publication bias was analyzed using Begg's funnel plot test.

\section{Results}

A total of 939 potential studies were collected from the database, as shown in Figure 1; 915 studies were removed because these articles were non-Beclin1-related, did not involve tests in tumor tissues, or were duplicated, as determined after their titles and abstracts were reviewed. A total of 24 studies were assessed by reading the full text; 13 studies were excluded because of the lack of cut-off values for Beclin1 expression 


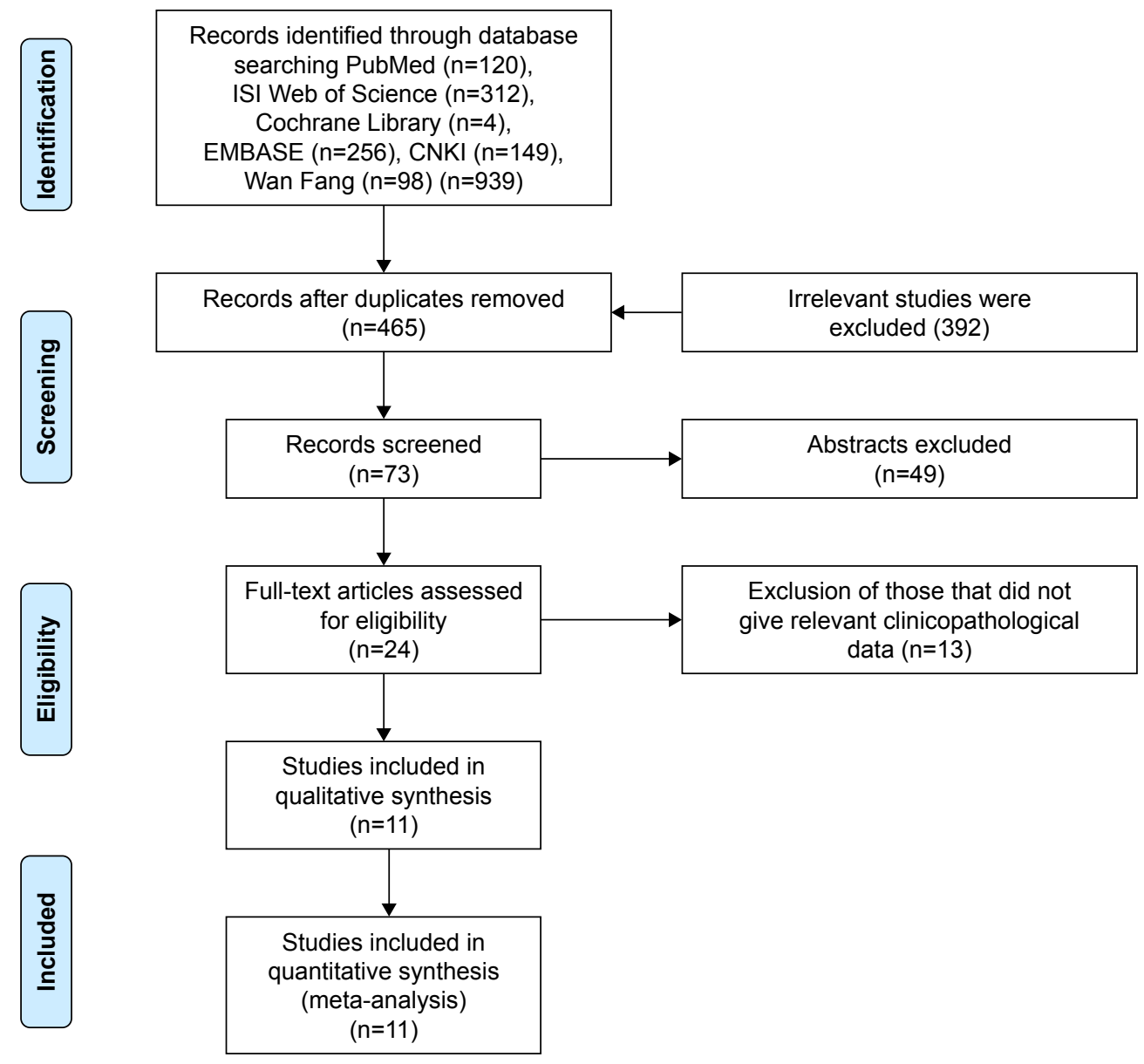

Figure I Flow diagram of study selection.

or insufficient information. Eleven ${ }^{14-24}$ eligible articles were ultimately included in this meta-analysis. Table 1 summarizes the characteristics of the articles. Published from 2007 to 2015 , these studies included a total of 1,279 patients with HCC. The sample sizes of these studies ranged from 43 to $300 ; 6$ studies included $\leq 100$ patients, and 5 studies included $>100$ patients. The 11 studies were from the following regions: 9 from People's Republic of China, 1 from Korea, and 1 from Egypt, Six of these studies were published in English, and the others were in Chinese. Each of these studies obtained a score $\geq 6$ in methodological assessment, which indicates high quality.

\section{Meta-analysis of clinicopathological characteristics}

The correlation between Beclin1 expression and the clinicopathological features of HCC was assessed. As shown in Table 2 and Figure 2, positive Beclin1 expression was negatively associated with alpha fetoprotein (AFP) $(\mathrm{OR}=0.58$, $95 \% \mathrm{CI}=[0.42,0.81], p=0.001$, fixed effect), liver cirrhosis
$(\mathrm{OR}=0.69,95 \% \mathrm{CI}=[0.48,0.99], p=0.045$, fixed effect $)$ and vascular invasion $(\mathrm{OR}=0.39,95 \% \mathrm{CI}=[0.18,0.85]$, $p=0.017$, random effect). However, Beclin1 expression was not significantly associated with gender $(\mathrm{OR}=0.80$, $95 \%$ CI $=[0.43,1.48], p=0.477$, random effect $)$, age (OR $=1.12,95 \% \mathrm{CI}=[0.84,1.49], p=0.444$, fixed effect $)$, HBsAg (OR $=1.13,95 \%$ CI $=[0.81,1.58], p=0.479$, fixed effect), tumor size (OR $=1.04,95 \% \mathrm{CI}=[0.91,1.19]$, $p=0.528$, fixed effect), tumor number (OR $=1.08,95 \%$ $\mathrm{CI}=[0.73,1.60], p=0.691$, fixed effect), differentiation $(\mathrm{OR}=0.64,95 \% \mathrm{CI}=[0.38,1.10], p=0.104$, random effect $)$, and TNM stage $(\mathrm{OR}=1.11,95 \% \mathrm{CI}=[0.81,1.54], p=0.438$, fixed effect).

As shown in Table 3, subgroup analysis of gender, vascular invasion, and differentiation was conducted using the sample size and the NOS score to explore the potential sources of heterogeneity. Notably, while the subgroups were classified by sample size, Beclin1 expression was correlated with vascular invasion $(\mathrm{n} \leq 100$ : $\mathrm{OR}=0.13,95 \% \mathrm{CI}=[0.05$, 0.34], $p=0.000$, fixed effect) in the subgroup with small 
Table I Characteristics of studies included in the meta-analysis

\begin{tabular}{|c|c|c|c|c|c|c|c|c|}
\hline Author & Country & $\begin{array}{l}\text { Tumor } \\
\text { type }\end{array}$ & $\begin{array}{l}\text { Number } \\
\text { of patients }\end{array}$ & $\begin{array}{l}\text { Laboratory } \\
\text { method }\end{array}$ & $\begin{array}{l}\text { Expression } \\
\text { Cancer }(+/-) \\
\text { Control }(+/-)\end{array}$ & $\begin{array}{l}\text { Gender } \\
\text { Male (+/-) } \\
\text { Female (+/-) }\end{array}$ & $\begin{array}{l}\text { Age } \\
>\mathbf{5 0}(+/-) \\
\leq \mathbf{5 0}(+/-)\end{array}$ & $\begin{array}{l}\text { HbsAg } \\
\text { Yes (+/-) } \\
\text { No (+/-) }\end{array}$ \\
\hline \multirow[t]{2}{*}{ Chen $^{17}$} & People's Republic & $\mathrm{HCC}$ & 76 & $\mathrm{IHC}$ & $64 / 12$ & $51 / 9$ & $13 / 2$ & $44 / 8$ \\
\hline & of China & & & & $22 / 52$ & $13 / 3$ & $51 / 10$ & $21 / 4$ \\
\hline \multirow[t]{2}{*}{ Ding et $\mathrm{al}^{18}$} & People's Republic & $\mathrm{HCC}$ & 300 & $\mathrm{IHC}$ & $95 / 205$ & $79 / 173$ & $53 / 103$ & $79 / 174$ \\
\hline & of China & & & & NA & $32 / 16$ & $42 / 102$ & $|6 / 3|$ \\
\hline \multirow[t]{2}{*}{ Guo et al ${ }^{19}$} & People's Republic & $\mathrm{HCC}$ & 54 & $\mathrm{IHC}$ & $44 / 10$ & $32 / 7$ & $29 / 7$ & NA \\
\hline & of China & & & & $55 / 0$ & $12 / 3$ & $15 / 3$ & \\
\hline \multirow[t]{2}{*}{ Lee et $\mathrm{a}^{20}$} & Korea & $\mathrm{HCC}$ & 190 & $\mathrm{IHC}$ & $11 / 179$ & $8 / 150$ & $2 / 91$ & $7 / 130$ \\
\hline & & & & & NA & $3 / 29$ & $9 / 88$ & $4 / 49$ \\
\hline \multirow[t]{2}{*}{ Kang et $\mathrm{a}^{21}$} & People's Republic & $\mathrm{HCC}$ & 50 & $\mathrm{IHC}$ & $39 / 11$ & NA & $27 / 7$ & $35 / 7$ \\
\hline & of China & & & & $25 / 45$ & & $12 / 4$ & $4 / 4$ \\
\hline \multirow[t]{2}{*}{ Liang $^{22}$} & People's Republic & $\mathrm{HCC}$ & 43 & $\mathrm{IHC}$ & $18 / 25$ & NA & $5 / 8$ & $18 / 20$ \\
\hline & of China & & & & $22 / 21$ & & $13 / 17$ & $0 / 5$ \\
\hline \multirow[t]{2}{*}{ Osman et al ${ }^{16}$} & Egypt & $\mathrm{HCC}$ & 65 & $\mathrm{IHC}$ & $33 / 32$ & $24 / 27$ & $9 / 12$ & $10 / 3$ \\
\hline & & & & & NA & $9 / 5$ & $24 / 20$ & $23 / 29$ \\
\hline \multirow[t]{2}{*}{ Qiu et al ${ }^{14}$} & People's Republic & $\mathrm{HCC}$ & 103 & $\mathrm{IHC}$ & $75 / 28$ & $62 / 23$ & $4 I / 12$ & $59 / 24$ \\
\hline & of China & & & & $5 I / 6$ & $13 / 5$ & $34 / 16$ & $15 / 4$ \\
\hline \multirow[t]{2}{*}{ Wu et al ${ }^{15}$} & People's Republic & $\mathrm{HCC}$ & 156 & $\mathrm{IHC}$ & $73 / 83$ & $69 / 74$ & $43 / 40$ & $59 / 58$ \\
\hline & of China & & & & NA & $4 / 9$ & $30 / 43$ & $14 / 25$ \\
\hline \multirow[t]{2}{*}{ Yang et $\mathrm{al}^{23}$} & People's Republic & $\mathrm{HCC}$ & 50 & $\mathrm{IHC}$ & $39 / 11$ & $31 / 8$ & $25 / 7$ & $25 / 7$ \\
\hline & of China & & & & $49 / 1$ & $8 / 3$ & $14 / 4$ & $14 / 4$ \\
\hline \multirow[t]{2}{*}{$\mathrm{He}^{24}$} & People's Republic & $\mathrm{HCC}$ & 192 & $\mathrm{IHC}$ & $19 / 173$ & $18 / 160$ & NA & $13 / 119$ \\
\hline & of China & & & & NA & $1 / 13$ & & $6 / 54$ \\
\hline
\end{tabular}

Abbreviations: AFP, alpha fetoprotein; DFS, disease-free survival; HCC, hepatocellular carcinoma; IHC, immunohistochemistry; NA, not applicable; OS, overall survival; UICC, Union for International Cancer Control.

sample size but not in the subgroup with a large sample size. Heterogeneity in differentiation was mainly observed in both subgroups $(\mathrm{n} \leq 100)\left(I^{2}=56.8 \%\right)(\mathrm{n}>100)\left(I^{2}=51.7 \%\right)$; however, heterogeneity in gender $(\mathrm{n}>100)\left(I^{2}=70.1 \%\right)$ and in vascular invasion $(\mathrm{n}>100)\left(I^{2}=62.9 \%\right)$ were only observed in the groups with a large sample size. On the basis of the NOS score, Beclin1 expression was correlated with vascular invasion $($ NOS score $\leq 7:$ OR $=0.21,95 \% \mathrm{CI}=[0.07,0.61]$, $p=0.004$, random effect). However, heterogeneity in both subgroups of differentiation and vascular invasion remained. These results indicated that heterogeneity in vascular invasion, tumor encapsulation, and TNM stage was most likely caused by the sample size and the NOS score.

\section{Meta-analysis of prognostic value}

We evaluated the prognostic value of Beclin1 in HCC. As shown in Figure 3, positive Beclin1 expression was associated with favorable 5-year OS $(\mathrm{HR}=0.68,95 \% \mathrm{CI}=[0.41,0.96]$, $p<0.00001$, fixed effect) and disease-free survival (DFS) (HR $=0.71,95 \% \mathrm{CI}=[0.51,0.92], p<0.00001$, fixed effect $)$.

\section{Publication bias}

As shown in Figures 3 and 4, no publication bias was found for gender $(p=0.406)$, age $(p=0.210)$, HBsAg $(p=0.210)$,
AFP $(p=0.403)$, liver cirrhosis $(p=0.536)$, tumor size $(p=0.1)$, number of tumors $(p=0.734)$, differentiation $(p=1)$, TNM stage $(p=0.386), 5$-year OS $(p=1)$, and DFS $(p=1)$, as determined by Begg's test. However, publication bias was observed for vascular invasion ( $p=0.035)$, as shown in Figures 3 and 4. Sensitivity analysis indicated that no study substantially influenced the pooled OR, indicating the need to include more studies in future research.

\section{Discussion}

Autophagy ${ }^{25,26}$ is a normal physiological process to maintain a sufficient level of intracellular homeostasis. It is regarded as a natural, regulatory, and destructive mechanism in the cell that allows cells to systematically recover from unwanted or malfunctioning groups. The process plays a key role in cell development for the provision of nutrients and energy for cells and has been closely associated with cancer development in recent years. As a component of the hVps34/III PI3K complex, Beclin1 plays a vital role in autophagy. ${ }^{27}$ The PI3K complex plays an important role in mediating the localization of other autophagocyanates in the precursor cell membrane, and cells die because of excessive self-loss during sustained stress and continuous progression of autophagy. The death of these cells is often characterized by autophagy; mainly the 


\begin{tabular}{|c|c|c|c|c|c|c|c|c|}
\hline $\begin{array}{l}\text { Liver } \\
\text { cirrhosis } \\
\text { Yes (+/-) } \\
\text { No (+/-) }\end{array}$ & $\begin{array}{l}\text { AFP } \\
\text { Positive (+/-) } \\
\text { Negative (+/-) }\end{array}$ & $\begin{array}{l}\text { Tumor } \\
\text { size } \\
\leq 5 \mathrm{~cm}(+/-) \\
>5 \mathrm{~cm}(+/-)\end{array}$ & $\begin{array}{l}\text { Tumor } \\
\text { number } \\
\text { Single }(+/-) \\
\text { Multiple }(+/-)\end{array}$ & $\begin{array}{l}\text { Vascular } \\
\text { invasion } \\
\text { Yes (+/-) } \\
\text { No (+/-) }\end{array}$ & $\begin{array}{l}\text { Differentiation } \\
\text { Low }(+/-) \\
\text { High and } \\
\text { Moderate }(+/-)\end{array}$ & $\begin{array}{l}\text { UICC stage } \\
\text { I, II (+/-) } \\
\text { III, IV (+/-) }\end{array}$ & Survival & $\begin{array}{l}\text { Quality } \\
\text { score }\end{array}$ \\
\hline $57 / 11$ & $35 / 7$ & $28 / 5$ & NA & $9 / 5$ & NA & $27 / 7$ & $N$ & 7 \\
\hline $7 / 1$ & $29 / 5$ & $36 / 7$ & & $55 / 7$ & & $35 / 5$ & & \\
\hline $84 / 191$ & $38 / 116$ & $53 / 110$ & $82 / 173$ & $12 / 29$ & $72 / 140$ & $70 / 149$ & OS/DFS & 8 \\
\hline $11 / 14$ & $57 / 89$ & $42 / 95$ & $13 / 32$ & $83 / 176$ & $23 / 65$ & $25 / 56$ & & \\
\hline $16 / 3$ & $17 / 4$ & $18 / 4$ & NA & $11 / 9$ & $21 / 2$ & $20 / 5$ & $\mathrm{~N}$ & 7 \\
\hline $28 / 7$ & $27 / 6$ & $26 / 6$ & & $33 / 1$ & $23 / 8$ & $28 / 1$ & & \\
\hline $8 / 88$ & $1 / 55$ & $7 / 107$ & NA & NA & $8 / 146$ & $6 / 74$ & $N$ & 8 \\
\hline $3 / 91$ & $10 / 124$ & $4 / 72$ & & & $3 / 33$ & $5 / 105$ & & \\
\hline \multirow[t]{2}{*}{ NA } & NA & $11 / 8$ & NA & NA & $18 / 8$ & NA & $N$ & 8 \\
\hline & & $28 / 3$ & & & $21 / 3$ & & & \\
\hline \multirow[t]{2}{*}{ NA } & $9 / 8$ & $7 / 12$ & NA & NA & $4 / 6$ & NA & $\mathrm{N}$ & 6 \\
\hline & $9 / 17$ & $1 \mathrm{I} / \mathrm{I3}$ & & & $14 / 19$ & & & \\
\hline $24 / 29$ & NA & $25 / 19$ & $22 / 21$ & NA & $8 / 17$ & $11 / 7$ & $N$ & 8 \\
\hline $9 / 3$ & & $8 / 13$ & $11 / 11$ & & $25 / 15$ & $22 / 25$ & & \\
\hline $58 / 25$ & $44 / 20$ & NA & $56 / 17$ & $18 / 15$ & $34 / 20$ & $8 / 2$ & OS & 9 \\
\hline $17 / 3$ & $31 / 8$ & & $19 / 11$ & $57 / 13$ & $4 I / 8$ & $67 / 26$ & & \\
\hline $53 / 59$ & NA & $44 / 41$ & $45 / 56$ & $19 / 16$ & $62 / 75$ & $37 / 35$ & OS/DFS & 8 \\
\hline $20 / 24$ & & $29 / 42$ & $28 / 27$ & $54 / 67$ & $11 / 8$ & $36 / 48$ & & \\
\hline $36 / 10$ & $14 / 4$ & $25 / 7$ & NA & $10 / 8$ & $17 / 9$ & NA & $N$ & 6 \\
\hline $3 / 1$ & $25 / 7$ & $14 / 4$ & & $29 / 3$ & $22 / 2$ & & & \\
\hline $14 / 143$ & $9 / 132$ & NA & NA & $6 / 74$ & $4 / 35$ & $5 / 47$ & OS/DFS & 7 \\
\hline $5 / 39$ & $10 / 41$ & & & $13 / 99$ & $15 / 138$ & $14 / 126$ & & \\
\hline
\end{tabular}

overexpression of Beclin1 and large amounts of autophagosomes produced by cells. ${ }^{28}$ The downregulation of Beclin1 protein expression in various tumors indicates that tumor development is closely related to Beclin1-induced autophagic cell death. ${ }^{12,13}$ Liang et $\mathrm{al}^{29}$ indicated that Beclin1 protein expression was significantly reduced or even undetectable in the breast cancer cell line MCF-7, and stable transfection of Beclin1 could significantly improve autophagy and reduce tumorigenic ability. In addition, the downregulation of the autophagy gene Beclin1 can significantly reduce autophagy to protect tumor cells from autophagic cell death, contributing to the continuous development of tumor cells. ${ }^{30}$

Genome instability is one of the hallmarks of tumor and is one of the important mechanisms of cell carcinogenesis. The instability of the cell genome is increased due to the environment of organism and metabolic stress. The monoallelic deletion of the autophagy gene Beclin1 can damage the cell autophagy ability and is not conducive to normal cells during metabolic stress survival, but promotes the occurrence of the tumor. ${ }^{31}$ Kung et $\mathrm{al}^{32}$ have demonstrated that

Table 2 Beclin I clinicopathological features for HCC

\begin{tabular}{|c|c|c|c|c|c|c|c|}
\hline \multicolumn{8}{|l|}{ Heterogeneity } \\
\hline $\begin{array}{l}\text { Clinicopathological } \\
\text { features }\end{array}$ & $\begin{array}{l}\text { Number } \\
\text { of studies }\end{array}$ & $\begin{array}{l}\text { Number } \\
\text { of patients }\end{array}$ & $\begin{array}{l}\text { Pooled OR } \\
(95 \% \mathrm{Cl})\end{array}$ & PHet & $I^{2}(\%)$ & $p$-value & $\begin{array}{l}\text { Model } \\
\text { used }\end{array}$ \\
\hline Gender & 9 & 1,186 & $0.80(0.43-1.48)$ & 0.028 & 53.4 & 0.477 & Random \\
\hline Age & 10 & $\mathrm{I}, 087$ & I.I2 (0.84-I.49) & 0.558 & 0.0 & 0.444 & Fixed \\
\hline $\mathrm{HbsAg}$ & 10 & 1,226 & $1.13(0.81-1.58)$ & 0.207 & 25.7 & 0.479 & Fixed \\
\hline AFP & 9 & 1,008 & $0.58(0.42-0.8 \mathrm{I})$ & 0.269 & 20.3 & 0.001 & Fixed \\
\hline Liver cirrhosis & 9 & $\mathrm{I}, 185$ & $0.69(0.48-0.99)$ & 0.129 & 36.2 & 0.045 & Fixed \\
\hline Tumor size & 9 & 984 & $1.04(0.91-1.19)$ & 0.343 & 11.0 & 0.528 & Fixed \\
\hline Vascular invasion & 7 & 931 & $0.39(0.18-0.85)$ & 0.001 & 72.1 & 0.017 & Random \\
\hline Tumor number & 4 & 624 & $1.08(0.73-1.60)$ & 0.476 & 0.0 & 0.691 & Fixed \\
\hline Differentiation & 10 & $\mathrm{I}, 203$ & $0.64(0.38-1.10)$ & 0.017 & 55.4 & 0.104 & Random \\
\hline TNM stage & 8 & $\mathrm{I}, 143$ & I.II (0.8I-I.54) & 0.487 & 0.00 & 0.438 & Fixed \\
\hline
\end{tabular}

Abbreviations: AFP, alpha fetoprotein; $\mathrm{Cl}$, confidence interval; Fixed, fixed-effects model; HCC, hepatocellular carcinoma; OR, odds ratio; Random, random-effects model. 
autophagic-deficient mouse models contain large amounts of damaged mitochondria, p62, and ubiquitin protein polymers. Massive accumulation of these substances can further induce reactive oxygen species production, leading

A



C

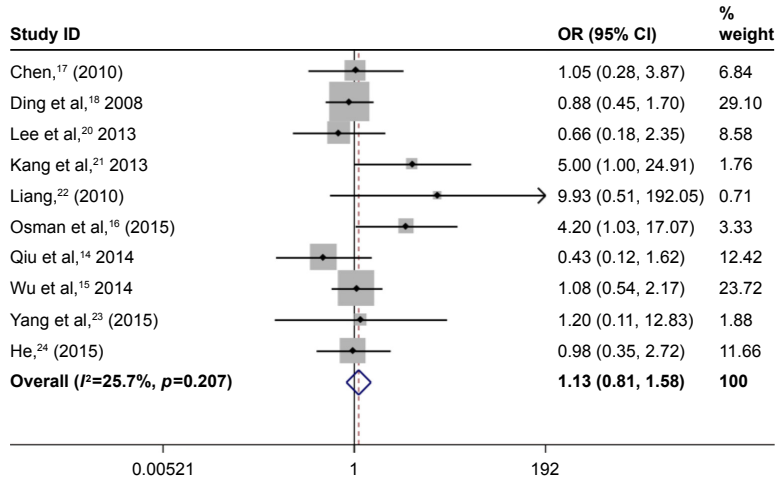

E



G

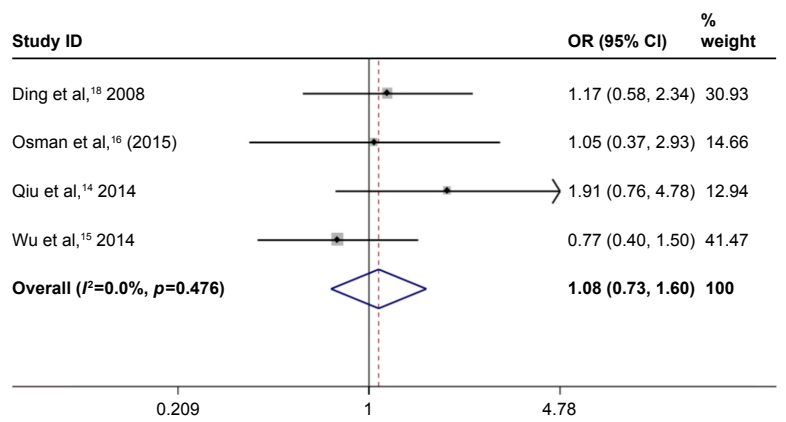

Figure 2 (Continued) to increased chromosomal instability, significantly increasing the incidence of cancer. Beclin1 can effectively prevent gene mutations, improve genomic stability, and ultimately reduce the incidence rate of cancer. However, the relevant

B

\begin{tabular}{|c|c|c|}
\hline Study ID & OR $(95 \% \mathrm{Cl})$ & $\begin{array}{l}\% \\
\text { weight }\end{array}$ \\
\hline Chen, ${ }^{17}(2010)$ & $1.27(0.25,6.54)$ & 3.04 \\
\hline Ding et al, ${ }^{18} 2008$ & $1.25(0.77,2.04)$ & 32.65 \\
\hline Guo et al,$^{19}(2013)$ & $0.83(0.19,3.67)$ & 4.40 \\
\hline Lee et al, ${ }^{20} 2013$ & $0.21(0.05,1.02)$ & 9.76 \\
\hline Kang et al, ${ }^{21} 2013$ & $1.29(0.32,5.24)$ & 3.80 \\
\hline Liang, ${ }^{22}(2010)$ & $0.82(0.22,3.09)$ & 5.48 \\
\hline Osman et al, ${ }^{16}(2015)$ & $0.62(0.22,1.78)$ & 10.03 \\
\hline Qiu et al, ${ }^{14} 2014$ & $1.61(0.67,3.86)$ & 8.97 \\
\hline Wu et al, ${ }^{15} 2014$ & $1.54(0.82,2.91)$ & 17.42 \\
\hline Yang et al, ${ }^{23}(2015)$ & $1.02(0.25,4.10)$ & 4.44 \\
\hline Overall $\left(l^{2}=0.0 \%, p=0.558\right)$ & $1.12(0.84,1.49)$ & 100 \\
\hline 0.0452 & 22.1 & \\
\hline
\end{tabular}

D

\begin{tabular}{|c|c|c|}
\hline Study ID & OR $(95 \% \mathrm{Cl})$ & $\begin{array}{l}\% \\
\text { weight }\end{array}$ \\
\hline Chen, ${ }^{17}(2010)$ & $0.74(0.08,6.63)$ & 2.95 \\
\hline Ding et al, ${ }^{18} 2008$ & $0.56(0.24,1.28)$ & 20.38 \\
\hline Guo et al, ${ }^{19}(2013)$ & $0.15(0.03,0.64)$ & 14.82 \\
\hline Lee et $\mathrm{al}_{,}{ }^{20} 2013$ & $2.76(0.71,10.73)$ & 4.04 \\
\hline Osman et al, ${ }^{16}$ (2015) & $0.28(0.07,1.13)$ & 11.69 \\
\hline Qiu et al, ${ }^{14} 2014$ & $0.41(0.11,1.52)$ & 12.01 \\
\hline Wu et al, ${ }^{15} 2014$ & $1.08(0.54,2.17)$ & 22.01 \\
\hline Yang et al, ${ }_{23}^{23}(2015)$ & $1.20(0.11,12.83)$ & 1.75 \\
\hline $\mathrm{He}_{,}{ }^{24}(2015)$ & $0.76(0.26,2.25)$ & 10.35 \\
\hline Overall $\left(I^{2}=36.2 \%, p=0.129\right)$ & $0.69(0.48,0.99)$ & 100 \\
\hline
\end{tabular}

F

Study ID

OR $(95 \% \mathrm{Cl}) \quad \stackrel{\%}{\text { weight }}$

Chen, ${ }^{17}(2010)$

Ding et al, ${ }^{18} 2008$

Guo et al, ${ }^{19}$ (2013)

Lee et $\mathrm{al}_{,}{ }^{20} 2013$

Kang et al, ${ }^{21} 2013$

Liang, ${ }^{22}$ (2010)

Osman et al, ${ }^{16}(2015)$

Wu et al, ${ }^{15} 2014$

Yang et al, ${ }^{23}$ (2015)

Overall $\left(I^{2}=11.0 \%, p=0.343\right)$

$1.01(0.83,1.23) 16.09$

$1.06(0.76,1.48) \quad 23.50$

$1.01(0.78,1.30) 10.91$

$1.17(0.35,3.85) 2.47$

$0.64(0.43,0.96) 10.96$

$0.80(0.39,1.67) 5.00$

$1.49(0.82,2.73) \quad 5.58$

$1.27(0.90,1.79) \quad 16.27$

$1.00(0.74,1.37) 9.23$
$1.04(0.91,1.19) \quad 100$

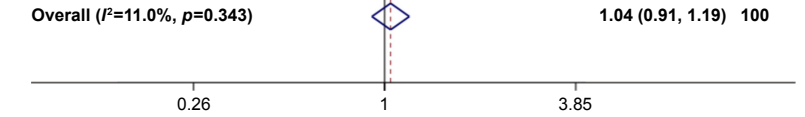

H

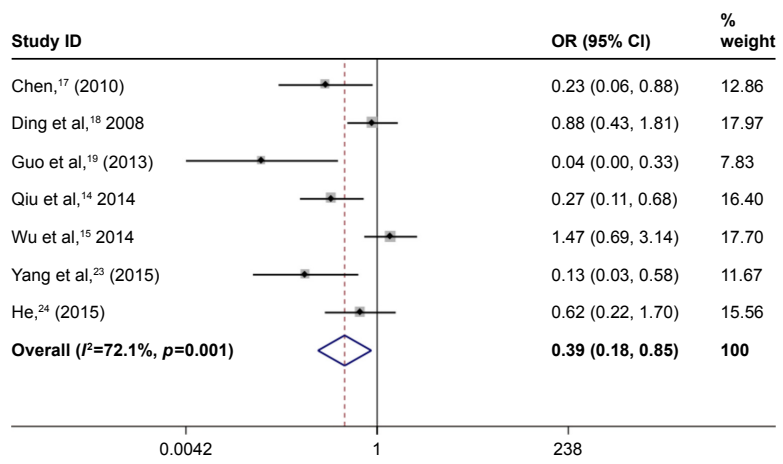




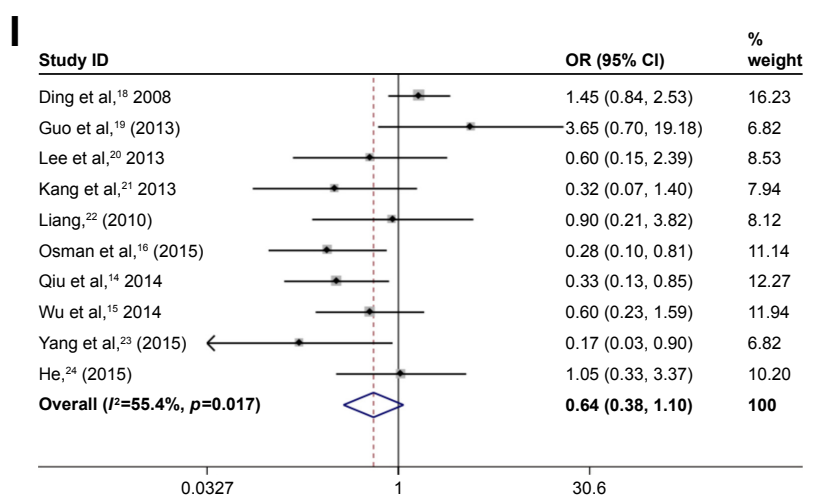

\begin{tabular}{|c|c|c|}
\hline Study ID & OR $(95 \% \mathrm{Cl})$ & $\begin{array}{l}\% \\
\text { weight }\end{array}$ \\
\hline Chen, ${ }^{17}(2010)$ & $0.55(0.16,1.93)$ & 9.34 \\
\hline Ding et al, ${ }_{, 18} 2008$ & $1.05(0.61,1.82)$ & 35.03 \\
\hline Guo et al, ${ }^{19}(2013) \leftarrow$ & $0.14(0.02,1.32)$ & 7.32 \\
\hline Lee et al, ${ }^{20} 2013$ & $1.70(0.50,5.79)$ & 5.49 \\
\hline Osman et al, ${ }^{16}(2015)$ & $1.79(0.59,5.40)$ & 6.68 \\
\hline Qiu et al, ${ }^{14} 2014$ & $1.55(0.31,7.80)$ & 3.67 \\
\hline Wu et al, ${ }^{15} 2014$ & $1.41(0.75,2.65)$ & 22.79 \\
\hline $\mathrm{He}{ }^{24}(2015)$ & $0.96(0.33,2.80)$ & 9.67 \\
\hline Overall $\left(I^{2}=0.0 \%, p=0.487\right)$ & $1.11(0.81,1.54)$ & 100 \\
\hline
\end{tabular}

Figure 2 Forest plot of studies evaluating the relationship between Beclin I expression and gender (A), age (B), HBsAg (C), liver cirrhosis (D), AFP (E), tumor size (F), number of tumors $(\mathbf{G})$, vascular invasion $(\mathbf{H})$, differentiation $(\mathbf{I})$, and TNM stage $(\mathbf{J})$.

Note: Weights are from random effects analysis.

Abbreviations: AFP, alpha fetoprotein; $\mathrm{Cl}$, confidence interval; OR, odds ratio.

mechanism may function as a conservative housekeeping mechanism to maintain genomic stability by reducing harmful proteins, removing damaged organelles, and reducing DNA damage..$^{33}$ In the stage of tumor development, the role of autophagy may have either positive or negative consequences: it can promote tumor cell proliferation, but it can also inhibit tumor cell growth, which is closely related to the state of the tumor itself..$^{34}$ Beclin1 has a proapoptotic function because of its BH3 domain. ${ }^{35}$ Thus, Beclin1 can inhibit tumor progression by promoting tumor cell apoptosis in tumor cells.
Recent studies have found abnormal Beclin1 expression in $\mathrm{HCC}$; however, results remain inconclusive. Lee et $\mathrm{al}^{20}$ have demonstrated that Beclin1 is not related to any clinicopathological features, such as the TNM stage and tumor size. Osman et $\mathrm{al}^{16}$ have found similar results. Qiu et al ${ }^{14}$ have indicated that Beclin1 expression in $\mathrm{HCC}$ is significantly lower than that in normal and cirrhotic tissues. They have also shown that Beclin1 expression is correlated with liver cirrhosis, Edmondson grade, and vascular invasion but not with gender, age, TNM stage, AFP level, number of tumors, and capsule integrity. In addition, low Beclin1 expression

Table 3 Subgroup analysis of tumor size and vascular invasion by sample size and NOS score

\begin{tabular}{|c|c|c|c|c|c|c|}
\hline Subgroups & $\begin{array}{l}\text { Number } \\
\text { of studies }\end{array}$ & $\begin{array}{l}\text { Number } \\
\text { of patients }\end{array}$ & $p$-value & $\begin{array}{l}\text { Pooled OR } \\
(95 \% \mathrm{Cl})\end{array}$ & PHet & $I^{2}(\%)$ \\
\hline \multicolumn{7}{|l|}{ Gender } \\
\hline \multicolumn{7}{|l|}{ Sample } \\
\hline $\mathrm{n} \leq 100$ & 4 & 344 & 0.863 & $0.94(0.46-1.90)$ & 0.650 & 0.0 \\
\hline$n>100$ & 5 & 842 & 0.531 & $0.73(0.28-1.94)$ & 0.010 & 70.1 \\
\hline \multicolumn{7}{|l|}{ NOS score } \\
\hline$\leq 7$ & 5 & 814 & 0.499 & $1.32(0.59-2.92)$ & 0.996 & 0.0 \\
\hline$>7$ & 4 & 372 & 0.269 & $0.62(0.26-1.45)$ & 0.015 & 67.5 \\
\hline \multicolumn{7}{|c|}{ Vascular invasion } \\
\hline \multicolumn{7}{|l|}{ Sample } \\
\hline $\mathrm{n} \leq 100$ & 3 & 372 & 0.000 & $0.13(0.05-0.34)$ & 0.363 & 1.4 \\
\hline $\mathrm{n}>100$ & 4 & 559 & 0.333 & $0.7 \mid(0.18-1.42)$ & 0.044 & 62.9 \\
\hline \multicolumn{7}{|l|}{ NOS score } \\
\hline$\leq 7$ & 4 & 368 & 0.004 & $0.2 \mathrm{I}(0.07-0.6 \mathrm{I})$ & 0.080 & 55.6 \\
\hline$>7$ & 3 & 563 & 0.500 & $0.73(0.29-1.81)$ & 0.019 & 74.6 \\
\hline \multicolumn{7}{|c|}{ Differentiation } \\
\hline \multicolumn{7}{|l|}{ Sample } \\
\hline $\mathrm{n} \leq 100$ & 5 & 262 & 0.195 & $0.64(0.38-1.10)$ & 0.055 & 56.8 \\
\hline $\mathrm{n}>100$ & 5 & 941 & 0.378 & $0.76(0.4 \mathrm{I}-\mathrm{I} .40)$ & 0.082 & 51.7 \\
\hline \multicolumn{7}{|l|}{ NOS score } \\
\hline$\leq 7$ & 6 & 339 & 0.837 & $0.89(0.30-2.65)$ & 0.084 & 54.9 \\
\hline$>7$ & 4 & 864 & 0.071 & $0.55(0.28-1.05)$ & 0.022 & 62.0 \\
\hline
\end{tabular}

Abbreviations: $\mathrm{Cl}$, confidence interval; NOS, Newcastle-Ottawa Scale; OR, odds ratio. 

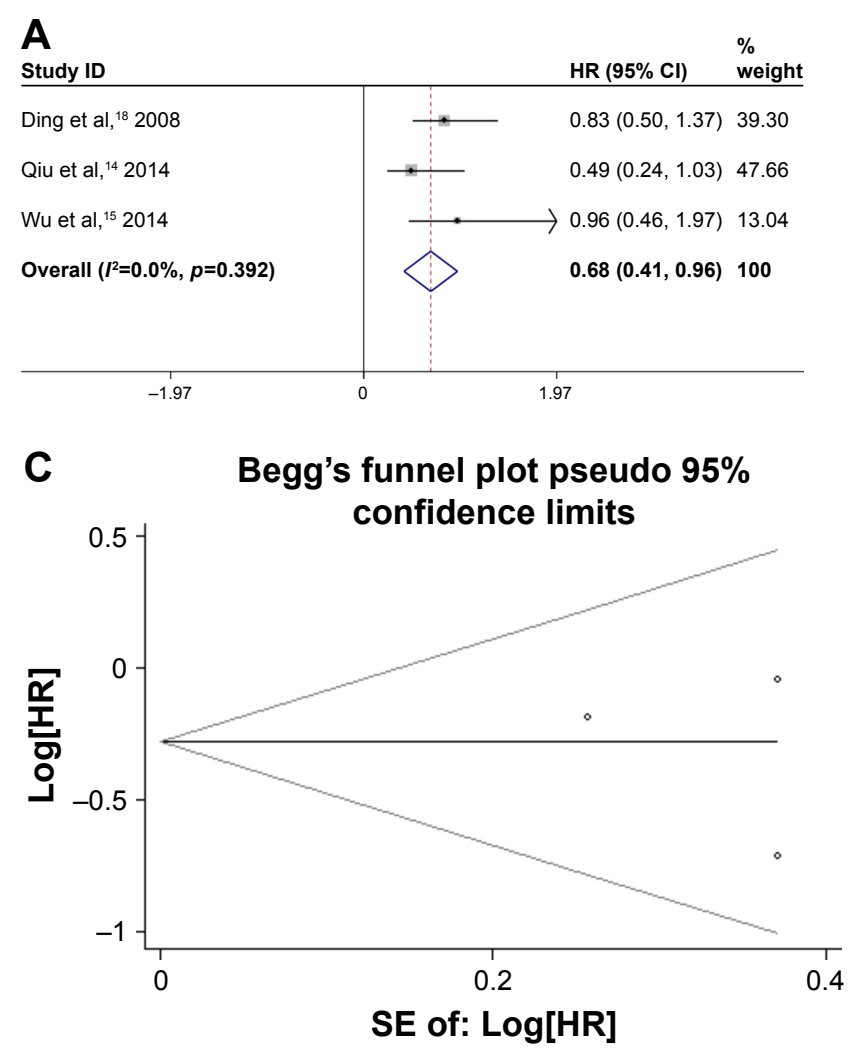
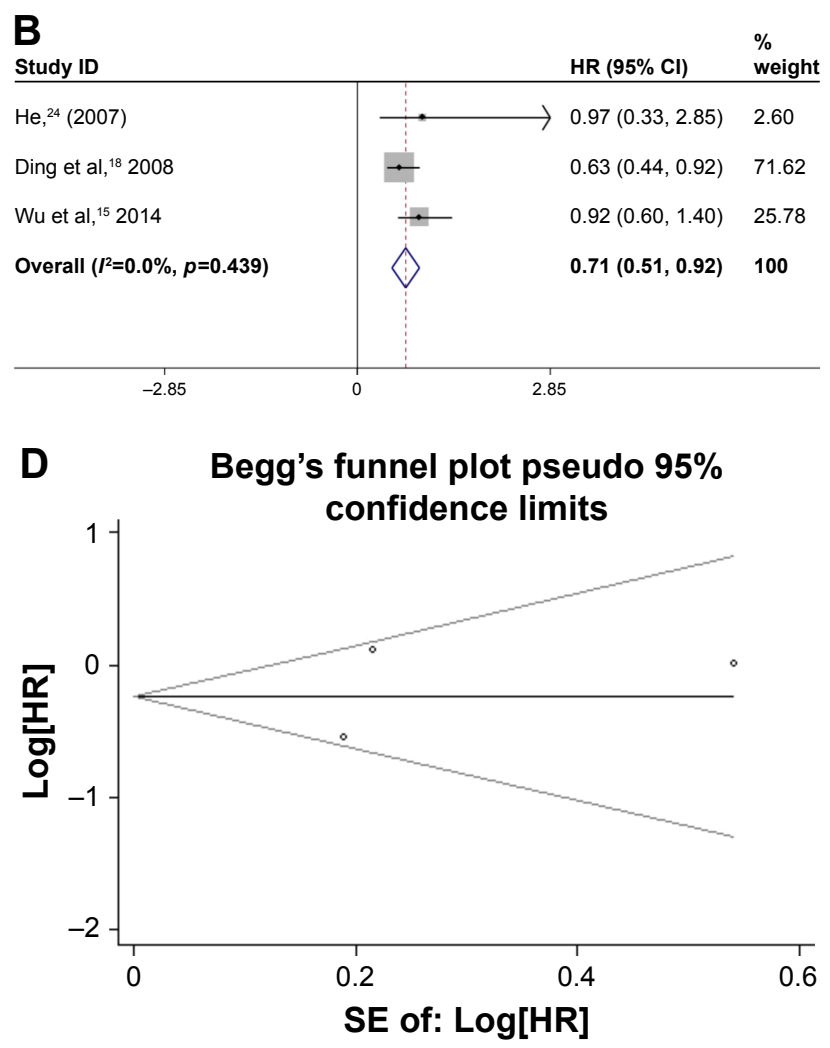

Figure 3 Pooled analysis for the association between Beclin I expression and 5-year survival rate (A: forest plots, C: Begg's publication bias plot), DFS rate (B: forest plots, D: Begg's publication bias plot).

Abbreviations: $\mathrm{Cl}$, confidence interval; DFS, disease-free survival; HR, hazard ratio; OR, odds ratio; SE, standard error.

indicates a poor OS. However, Wu et $\mathrm{al}^{15}$ have demonstrated that Beclin1 expression is not related to the clinical features and prognosis of HCC. In this meta-analysis, 1,279 patients from 11 articles were considered. Positive Beclin1 expression is associated with AFP, liver cirrhosis, and vascular invasion but not with gender, age, HBsAg, AFP, liver cirrhosis, tumor size, number of tumors, differentiation, and TNM stage. Eight studies with 1,008 patients are considered in this meta-analysis to explore the relationship between Beclin1 expression and AFP level. In 6 out of 8 studies, no relation was found between Beclin1 expression and the AFP level; however, the pooled results suggest a significant association between Beclin1 expression and the AFP level. Similarly, 8 of 9 studies and 3 of 7 studies have demonstrated that Beclin1 expression is not correlated with liver cirrhosis and vascular invasion; however, the pooled results indicate that Beclin1 expression is significantly related to liver cirrhosis and vascular invasion. In addition, we have indicated that positive Beclin1 expression can predict favorable 5-year survival and DFS rates. All pooled results have shown that Beclin1 is related to the development of HCC and that Beclin1 can be a potential prognostic biomarker for HCC.
In our meta-analysis, Beclin1 expression is only related to AFP, liver cirrhosis, and vascular invasion with respect to clinicopathological features; however, recent studies indicate that combined with other related genes, such as HIF- $1 \alpha$ and Bcl-xL, Beclin1 expression can considerably increase its clinical value. Ding et al ${ }^{18}$ have demonstrated that Beclin1 expression is significantly correlated with tumor differentiation in $\mathrm{Bcl}-\mathrm{xL}^{+}$patients but not in $\mathrm{Bcl}-\mathrm{xL}^{-}$patients. Moreover, positive Beclin1 expression indicates higher OS and DFS in Bcl-xL-positive HCC. Similar results have been found between Beclin1 and apoptotic markers, such as Bcl-2 and Bax. ${ }^{36,37}$ Osman et a ${ }^{16}$ have found that Beclin-1 expression is closely related to tumor differentiation, TNM stage, tumor size, and number of tumors in the high HIF- $1 \alpha$ expression group among patients with HCC but not in the low HIF-1 $\alpha$ expression group. Thus, Beclin1 combined with other molecules can be used to diagnose HCC and predict its prognosis.

Significant heterogeneity was determined in our study. Although the study included subgroup analysis and sensitivity analysis, the source of heterogeneity was not clearly identified. However, the heterogeneity may result from different factors. One is the laboratory method. Our meta-analysis 
included studies in which outcomes were assessed by IHC, a semiquantitative method, which can influence the quality of the antibody, incubation time, time of diaminobezidin staining, and so on. In addition, IHC has a subjective nature,

A

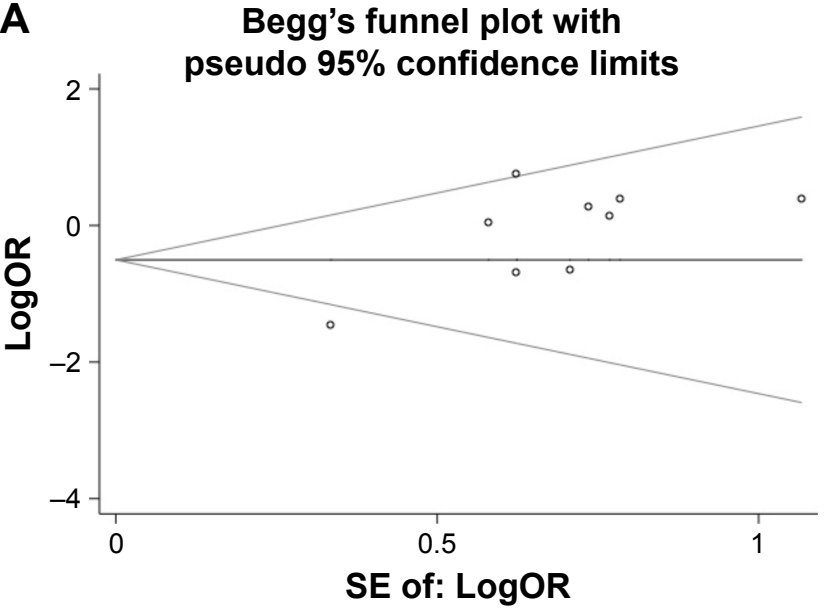

C

C Begg's funnel plot with

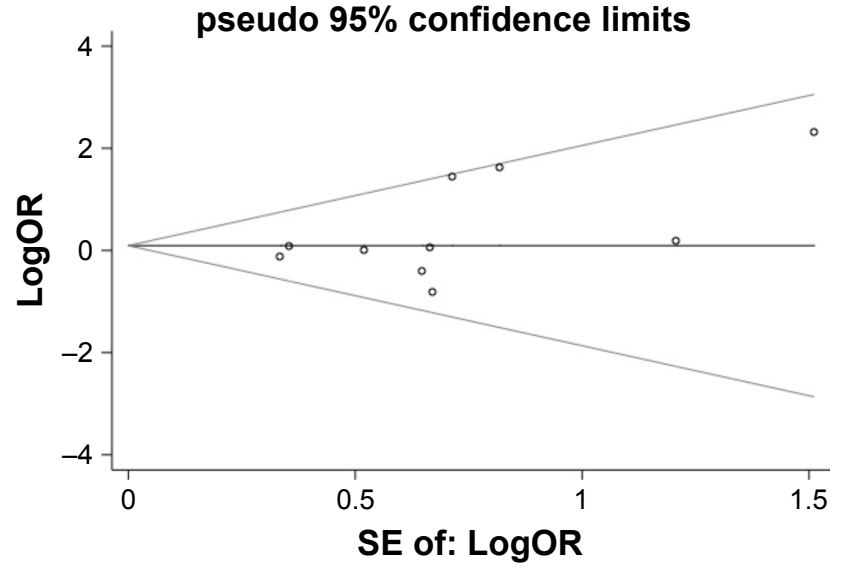

E Begg's funnel plot with

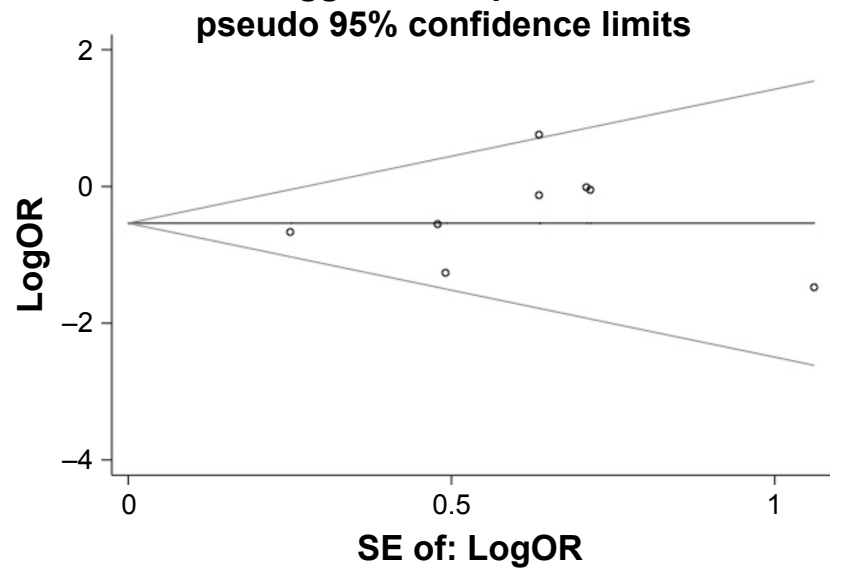

which may affect the evaluation. Another factor is the shortage of eligible articles for meta-analysis. High-quality, largescale studies are needed to analyze the relationship between Beclin1 and HCC.

B
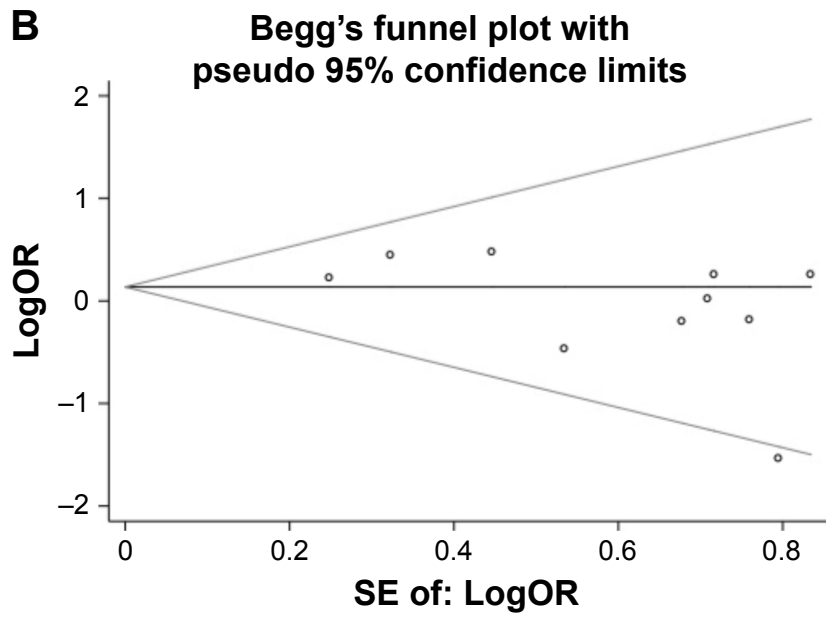

D Begg's funnel plot with

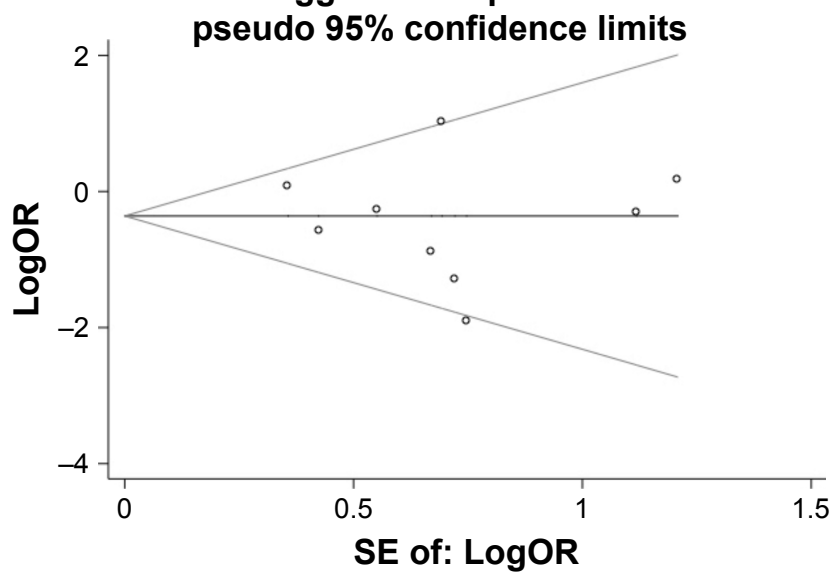

$\mathbf{F}$
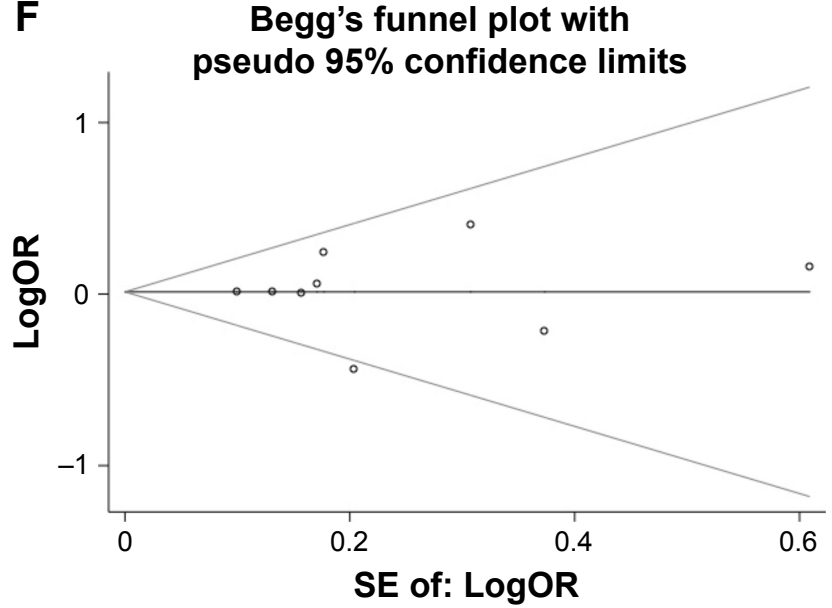

Figure 4 (Continued) 

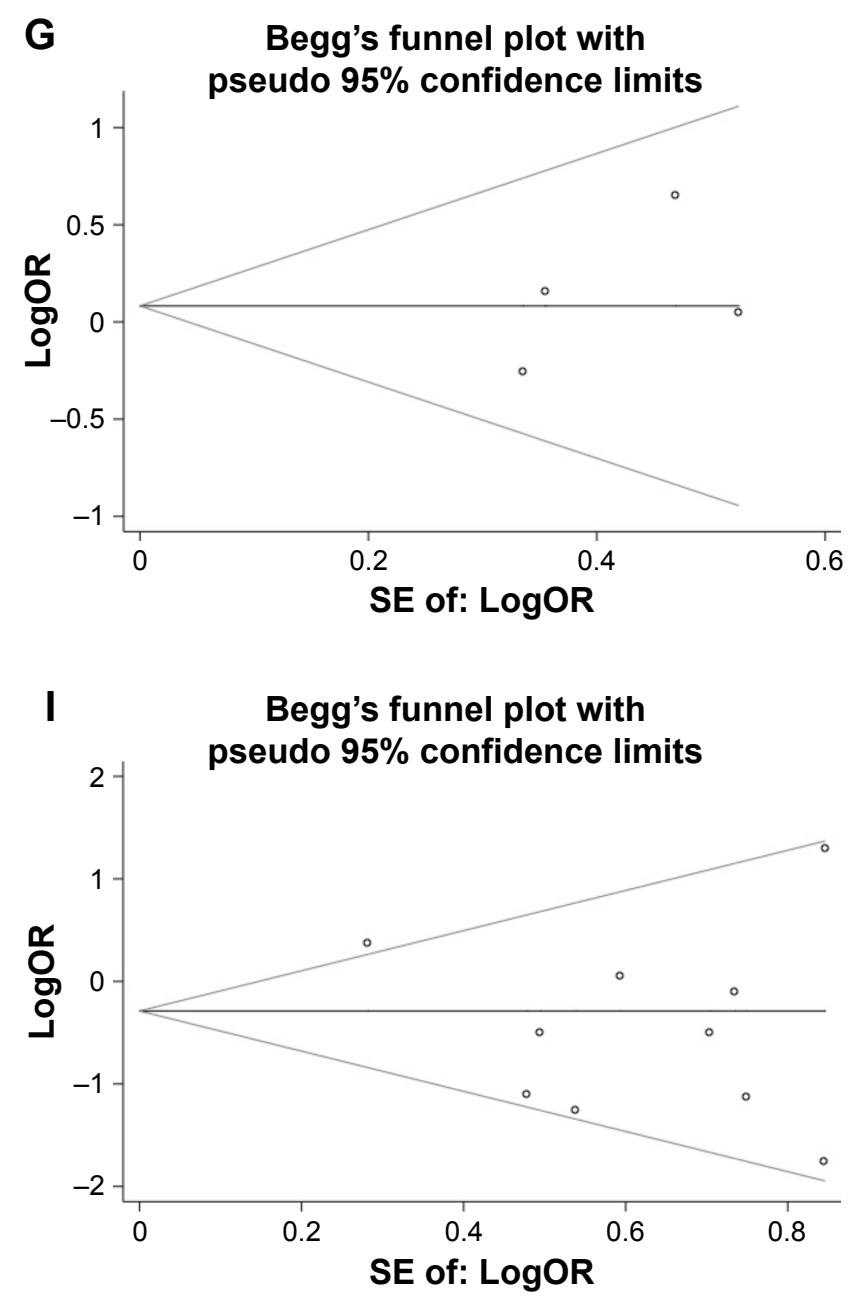
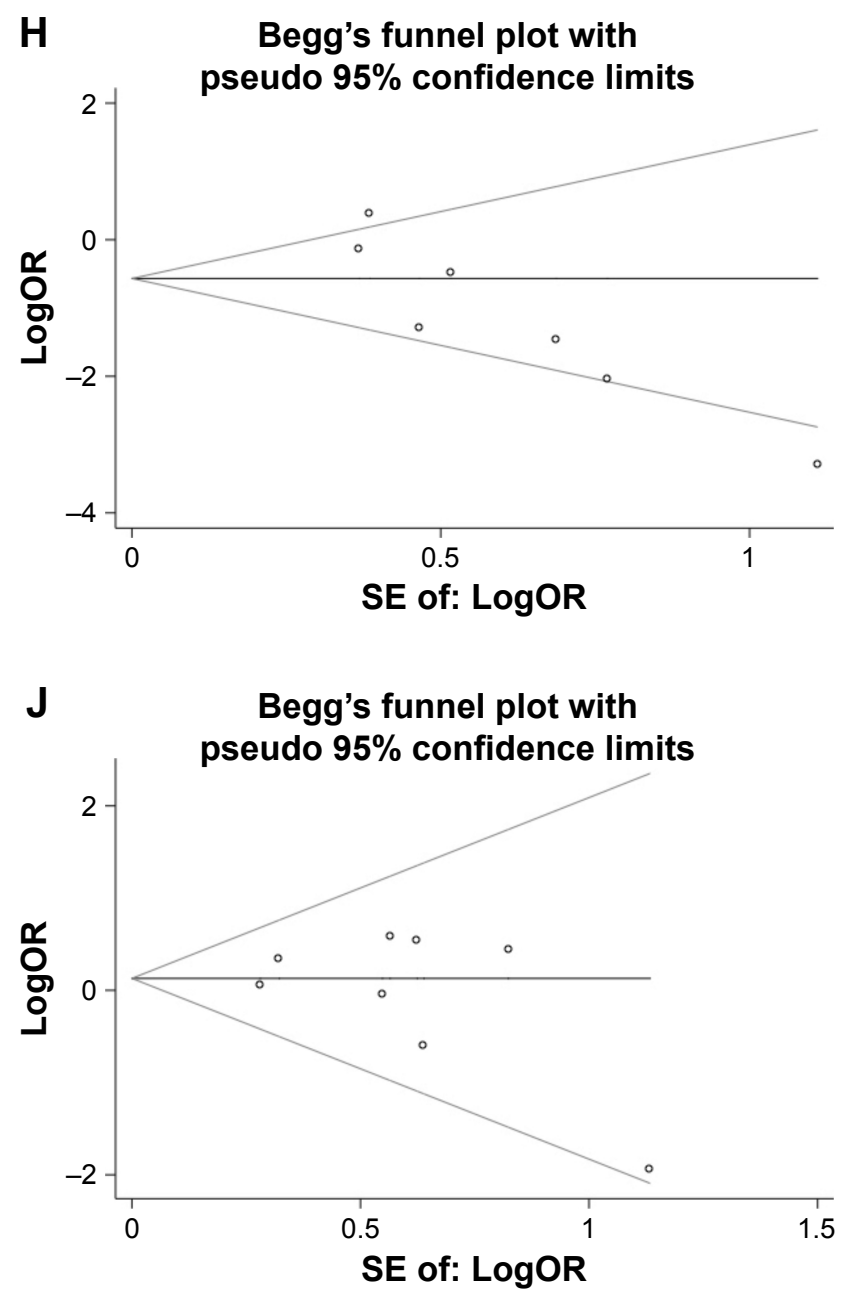

Figure 4 Begg's publication bias plot for Beclin l-related studies: gender (A), age (B), HBsAg (C), liver cirrhosis (D), AFP (E), tumor size (F), number of tumors (G), vascular invasion $(\mathbf{H})$, differentiation $(\mathbf{I})$, and TNM stage $(\mathbf{J})$.

\section{Limitations}

Our meta-analysis has certain limitations: 1) more studies are needed to support the current conclusions; 2) this metaanalysis mostly included studies that were conducted in the People's Republic of China, so that the pooled results may be applicable for Chinese or Asian populations only; thus, more studies from other regions or countries should be included to support the results; 3 ) bias may be present because of the HRs derived from the survival curves in the studies.

\section{Conclusion}

The results demonstrate that positive Beclin1 expression is negatively associated with AFP, liver cirrhosis, and vascular invasion in HCC. Moreover, Beclin1 may be used as a prognostic marker for HCC. However, these results should be verified by well-designed studies with a larger sample size.

\section{Acknowledgment}

This study received funding from Beijing Municipal Administration of Hospital Clinical Medicine Development of Special Funding Support (Grant no ZYLX201612) and Capital Foundation of Medical Development (shoufa20162-2053).

\section{Disclosure}

The authors report no conflicts of interest in this work.

\section{References}

1. Gong XL, Qin SK. Progress in systemic therapy of advanced hepatocellular carcinoma. World J Gastroenterol. 2016;22(29):6582-6594.

2. Zhu RX, Seto WK, Lai CL, Yuen MF. Epidemiology of hepatocellular carcinoma in the Asia-Pacific Region. Gut Liver. 2016;10(3): 332-339.

3. Forner A, Llovet JM, Bruix J. Hepatocellular carcinoma. Lancet. 2012;379(9822):1245-1255.

4. Avila MA, Berasain C, Sangro B, Prieto J. New therapies for hepatocellular carcinoma. Oncogene. 2006;25(27):3866-3884.

5. Nishikawa H, Kimura T, Kita R, Osaki Y. Treatment for hepatocellular carcinoma in elderly patients: a literature review. J Cancer. 2013;4(8): 635-643.

6. Lee YJ, Jang BK. The role of autophagy in hepatocellular carcinoma. Int J Mol Sci. 2015;16(11):26629-26643.

7. Yu J, Lan L, Lewin SJ, et al. Identification of novel small molecule Beclin 1 mimetics activating autophagy. Oncotarget. 2017;8(31): 51355-51369. 
8. Geng QR, Xu DZ, He LJ, et al. Beclin-1 expression is a significant predictor of survival in patients with lymph node-positive gastric cancer. PLoS One. 2012;7(9):e45968.

9. Ahn $\mathrm{CH}$, Jeong EG, Lee JW, et al. Expression of beclin-1, an autophagy-related protein, in gastric and colorectal cancers. APMIS. 2007;115(12):1344-1349.

10. Shen MX, Ding JB. Expression levels and roles of EMC-6, Beclin1, and Rab5a in the cervical cancer. Eur Rev Med Pharmacol Sci. 2017; 21(13):3038-3046.

11. Gu Y, Chen T, Li G, et al. Lower beclin 1 downregulates HER2 expression to enhance tamoxifen sensitivity and predicts a favorable outcome for ER positive breast cancer. Oncotarget. 2017;8:52156-52177.

12. Xia P, Wang JJ, Zhao BB, Song CL. The role of beclin-1 expression in patients with gastric cancer: a meta-analysis. Tumour Biol. 2013; 34(6):3303-3307.

13. Han Y, Xue XF, Shen HG, et al. Prognostic significance of Beclin-1 expression in colorectal cancer: a meta-analysis. Asian Pac J Cancer Prev. 2014;15(11):4583-4587.

14. Qiu DM, Wang GL, Chen L, et al. The expression of beclin-1, an autophagic gene, in hepatocellular carcinoma associated with clinical pathological and prognostic significance. BMC Cancer. 2014; $14: 327$.

15. Wu DH, Jia CC, Chen J, et al. Autophagic LC3B overexpression correlates with malignant progression and predicts a poor prognosis in hepatocellular carcinoma. Tumour Biol. 2014;35(12):12225-12233.

16. Osman NA, Abd El-Rehim DM, Kamal IM. Defective beclin-1 and elevated hypoxia-inducible factor (HIF)-1 $\alpha$ expression are closely linked to tumorigenesis, differentiation, and progression of hepatocellular carcinoma. Tumour Biol. 2015;36(6):4293-4299.

17. Chen X. Expression and Clinical Significance of Akt2 and Beclin1 in Primary Hepatic Carcinoma [master's thesis]. Suzhou, People's Republic of China: Soochow University; 2010.

18. Ding ZB, Shi YH, Zhou J, et al. Association of autophagy defect with a malignant phenotype and poor prognosis of hepatocellular carcinoma. Cancer Res. 2008;68(22):9167-9175.

19. Guo X, Yuejuan G, Wei W, et al. Expression and clinical significance of beclin1 in patients with hepatocellular carcinoma. Prog Modern Biomed. 2013;13:85-87.

20. Lee YJ, Hah YJ, Kang YN, et al. The autophagy-related marker LC3 can predict prognosis in human hepatocellular carcinoma. PLoS One. 2013;8(11):e81540.

21. Kang KF, Wang XW, Chen XW, et al. Beclin 1 and nuclear factorкBp65 are upregulated in hepatocellular carcinoma. Oncol Lett. 2013; 5(6):1813-1818.
22. Liang Q, Expression and Clinical Significance of Autophagy-related Genes Beclin1 in Hepatocellular Carcinoma [thesis]. Nanning: Guangxi Medical University; 2010.

23. Yang W, Hongjie Y, Ruixiang M, et al. The expression of Beclin1 in hepatocellular carcinoma and the effect of TGF- $\beta$ on proliferation of hepatoma cells [thesis]. Int J Lab Med. 2015;36:108-110.

24. He J. The Expression of Apoptosis and Autophagy Genes in Hepatocellular Carcinoma and its Clinical Implications [thesis]. Shanghai: Fudan University; 2007.

25. Gomes LC, Odedra D, Dikic I, Pohl C. Autophagy and modular restructuring of metabolism control germline tumor differentiation and proliferation in C. elegans. Autophagy. 2016;12(3):529-546.

26. Nakatogawa H, Ohsumi Y. Autophagy: close contact keeps out the uninvited. Curr Biol. 2014;24(12):R560-R562.

27. Kihara A, Kabeya Y, Ohsumi Y, Yoshimori T. Beclin-phosphatidylinositol 3-kinase complex functions at the trans-Golgi network. EMBO Rep. 2001;2(4):330-335.

28. Sinha S, Levine B. The autophagy effector Beclin 1: a novel BH3-only protein. Oncogene. 2008;27(Suppl 1):S137-S148.

29. Liang XH, Jackson S, Seaman M, et al. Induction of autophagy and inhibition of tumorigenesis by beclin 1. Nature. 1999;402(6762):672-676.

30. Wang ZH, Xu L, Duan ZL, Zeng LQ, Yan NH, Peng ZL. Beclin 1-mediated macroautophagy involves regulation of caspase-9 expression in cervical cancer HeLa cells. Gynecol Oncol. 2007;107(1): 107-113.

31. Westcott PM, Halliwill KD, To MD, et al. The mutational landscapes of genetic and chemical models of Kras-driven lung cancer. Nature. 2015;517(7535):489-492.

32. Kung CP, Budina A, Balaburski G, Bergenstock MK, Murphy M. Autophagy in tumor suppression and cancer therapy. Crit Rev Eukaryot Gene Expr. 2011;21(1):71-100.

33. Liang S, Figtree G, Aiqun M, Ping Z. GAPDH-knockdown reduce rotenone-induced $\mathrm{H} 9 \mathrm{C} 2$ cells death via autophagy and anti-oxidative stress pathway. Toxicol Lett. 2015;234(3):162-171.

34. Levy JMM, Towers CG, Thorburn A. Targeting autophagy in cancer. Nat Rev Cancer. 2017;17(9):528-542.

35. Levine B, Sinha S, Kroemer G. Bcl-2 family members: dual regulators of apoptosis and autophagy. Autophagy. 2008;4(5):600-606.

36. Al-Shenawy HA. Expression of Beclin-1, an autophagy-related marker, in chronic hepatitis and hepatocellular carcinoma and its relation with apoptotic markers. APMIS. 2016;124(3):229-237.

37. Kotsafti A, Farinati F, Cardin R, Cillo U, Nitti D, Bortolami M. Autophagy and apoptosis-related genes in chronic liver disease and hepatocellular carcinoma. BMC Gastroenterol. 2012;12:118.
OncoTargets and Therapy

\section{Publish your work in this journal}

OncoTargets and Therapy is an international, peer-reviewed, open access journal focusing on the pathological basis of all cancers, potential targets for therapy and treatment protocols employed to improve the management of cancer patients. The journal also focuses on the impact of management programs and new therapeutic agents and protocols on

\section{Dovepress}

patient perspectives such as quality of life, adherence and satisfaction. The manuscript management system is completely online and includes a very quick and fair peer-review system, which is all easy to use. Visit http://www.dovepress.com/testimonials.php to read real quotes from published authors. 